\title{
Italy's largest hole in ground
}

ITALIAN high-energy physics is now the proud possessor of the largest artificial subterranean cavity in the world. The objective is to mount a refined measurement of proton decay. But how this will be done will be decided only after an international meeting to be held in Rome next April.

The $100,000-\mathrm{m}^{3}$ cavern, 4,000 metres under the Gran Sasso in the Apennines, is a by-product of the construction of what is claimed to be the largest pair of road tunnels in the world, linking Rome by autostrada to the Adriatic coast. The cavern has cost the Italian physics budget nothing, but has been built at a cost of 80,000 million lira ( $£ 32$ million) by the Ministry of Public Works, partly as a means of providing jobs. But now it must be equipped, at a cost estimated at 80,000 100,000 lira.

The Gran Sasso experiment could become the most advanced proton decay measurement in the world, if only the money for instrumentation is forthcoming. According to one British physicist involved in another proton lifetime experiment, the Italian plans are "very futuristic" and the physicists may be "slightly embarrassed by the size of their hole". Proton decay is one of the testable predictions of "grand unified theories" linking all fundamental forces except gravity in a single theory; the existence of magnetic monopoles is one of many others.

Professor Antonino Zichichi, the expresident of the Italian institute for nuclear physics (INFN), who planned the Gran Sasso hall, says that while some of the cost will be met from Italian sources, international support from Europe and the United States will also be necessary. Next April's meeting will debate the nature of the equipment to be installed in Gran Sasso, and specifically whether it should be 10,000 tons of water (to detect decay products such as pions, which travel faster than the speed of light in the medium, by their Cerenkov radiation), or some other form of particle detector.

Water is the cheapest solution, but another 10,000-ton water experiment has been under way in a salt-mine under Lake Michigan for a year without detecting any definite decays. The simplest grand unified theory is now generally considered ruled out by the Michigan result. So it may be necessary to equip Gran Sasso to detect, for example, slow kaons (too slow to make Cerenkov light in water) which result from proton decay in the more sophisticated grand unified theories now favoured.

Other proton decay experiments under way or planned in this area are:

- An Italian measurement 5,000 metres under Mont Blanc, which has been running since 1981 but with a small (150 ton) detector mass. After the Michigan result, it now seems that the detectable lifetime of the proton will be beyond reach of this experiment, although it has recorded one unexplained event.

- An experiment in the Kolar gold mine in India, of similar scale and age, which claims three unexplained events.

- A Franco-German experiment in the Fréjus rail tunnel 4,900 metres under the Alps, between Turin and Lyon, using $1,000-1,500$ tons of water crossed by thousands of detector-sandwiches. Although this experiment uses water, it may be able to detect kaons through decay products of the kaons themselves.

- A 1,000-ton calorimetric US-British
High-energy physics

\section{Italian budget to be doubled}

THE Italian institute for nuclear research (INFN), which supports most of Italy's basic research in high- and low-energy nuclear physics, is likely to have its budget for 1985 more than doubled, to a total of 194,400 million lira ( $£ 85$ million). This sum excludes the 50,000-million lira subscription to CERN, the international organization for nuclear research near Geneva.

This increase is a mark of the Italian government's will to build on Italy's important position in world high-energy physics, attested by Carlo Rubbia's recent Nobel Prize for the discovery of the $W$ and $\mathrm{Z}$ particles. The increase will pay for Italian participation in HERA, the German electron-proton collider now under construction in Hamburg, a large proton lifetime experiment under the Apennines and other major projects including a study of a $50-\mathrm{TeV}$ proton-antiproton collider (the ELOISATRON) which might be built in southern Italy.

This expansion in Italian high-energy physics makes a telling contrast with the falling support for nuclear and high-energy physics in countries such as Britain, where domestic spending has fallen by 55 per cent over the past decade. Moreover, the Italians have a lesson for the British; the problem lies with the physicists, who have failed to make their subject popular, and not with the politicians, says the now expresident of INFN, Professor Antonino Zichichi. After "tremendous efforts" to popularize particle physics in print, in person, in theatres all around the country and on television, in flamboyant exercises which have sometimes brought him scorn among the less adventurous, Zichichi has fired the Italian imagination and won support for the first three years of his ambitious five-year plan for INFN, proposed more than a year ago. "You must convince the people first", says Zichichi, "and the politicians will follow". experiment that is under construction 1,800 metres deep in an iron mine in Minnesota.

Dr Wade Allison of the University of Oxford, who helped design the detector, says this Minnesota equipment will be well able to detect kaon decay modes, and that it may not be necessary to build detectors as big as 10,000 tons to get interesting results. All existing detectors are finding "inexplicable" events at the rate of 10 per year per 1,000 tons of detector, he says. These events are isolated in the chamber and do not, on the face of it, seem to be proton decays. But he suggests that they may be strange interactions of neutrinos emanating from cosmic-ray showers in the upper atmosphere or even experimental artefacts, but that the Minnesota experiment should decide.

Robert Walgate

This is why, Zichichi claims, the Italian parliament's budget committee and the lower house have already approved his plan. The senate is expected to follow soon. The figures give INFN 194,400 million lira next year, 220,000 million in 1986 and 230,000 million in 1987 , even above the rate implied by the 1 million million total requested in the five-year plan.

INFN's present president, theorist Nicola Cabbibo, is cautiously awaiting the

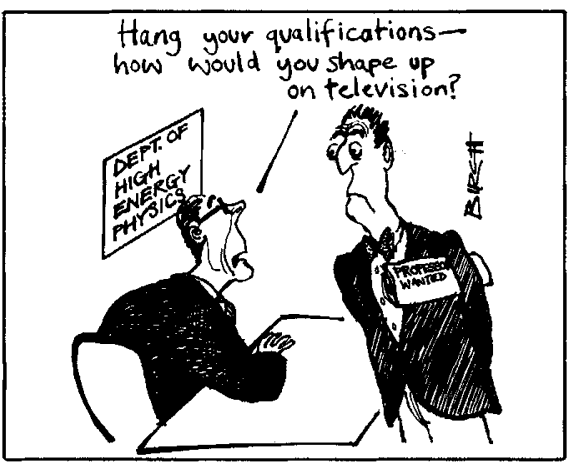

senate's decision before making any statement, but Zichichi considers the senate vote a formality.

Apart from rousing popular support for high-energy physics, Zichichi also claims to have shown politicians the benefit of industrial involvement. Italian companies will be building many of the superconducting magnets and some of the electronics for the German HERA, thus putting Italy in a good position in a field where the technology is changing rapidly and industrial applications cannot be far off. The proton lifetime and other experiments in an artificial cavern under the mountain Gran Sasso has also provided employment in a region where jobs are scarce. During his campaign for a bigger budget, Zichichi has zealously exploited all these sideeffects of high-energy physics. To those who have laughed at him, it seems he can now laugh back.
Robert Walgate 